\title{
Dynamics and Structural Changes Induced by ATP Binding in SAV1866, a Bacterial ABC Exporter
}

\author{
Jean-Paul Becker, ${ }^{\dagger}$ Françoise Van Bambeke, ${ }^{\ddagger}$ Paul M. Tulkens, ${ }^{\ddagger}$ and Martine Prévost ${ }^{*}, \dagger$ \\ Structure et Fonction des Membranes Biologiques, Université Libre de Bruxelles, Boulevard du Triomphe CP \\ 206/2, B-1050 Brussels, Belgium, and Unité de Pharmacologie cellulaire et moléculaire, Université catholique \\ de Louvain, Avenue E. Mounier 73, B-1200 Brussels, Belgium
}

Received: April 28, 2010; Revised Manuscript Received: September 6, 2010

\begin{abstract}
Multidrug transporters of the ATP-binding cassette family export a wide variety of compounds across membranes in both prokaryotes and eukaryotes, using ATP hydrolysis as energy source. Several of these membrane proteins are of clinical importance. Although biochemical and structural studies have provided insights into the mechanism underlying substrate transport, many key questions subsist regarding the molecular and structural nature of this mechanism. In particular, the detailed conformational changes occurring during the catalytic cycle are still elusive. We explored the conformational changes occurring upon ATP/Mg ${ }^{2+}$ binding using molecular dynamics simulations starting from the nucleotide-bound structure of SAV1866 embedded in an explicit lipid bilayer. The removal of nucleotide revealed a major rearrangement in the outer membrane leaflet portion of the transmembrane domain (TMD) resulting in the closure of the central cavity at the extracellular side. This closure is similar to that observed in the crystal nucleotide-free structures. The interface of the nucleotide-binding domain dimer (NDB) is significantly more hydrated in the nucleotide-free trajectory though it is not disrupted. This finding suggests that the TMD closure could occur as a first step preceding the dissociation of the dimer. The transmission pathway of the signal triggered by the removal of $\mathrm{ATP} / \mathrm{Mg}^{2+}$ mainly involves the conserved Q-loop and X-loop as well as TM6.
\end{abstract}

\section{Introduction}

ATP-binding cassette $(\mathrm{ABC})$ proteins form a large family in both prokaryotes and eukaryotes. Its members carry a wide range of structurally diverse compounds across cell membranes: polypeptides, sugars, lipids, ions, vitamins, and hydrophobic compounds. Two subtypes of ABC transporters are defined according to the direction of solute transport: $\mathrm{ABC}$ importers, present only in prokaryotes, and $\mathrm{ABC}$ exporters. $\mathrm{ABC}$ transporters are of biomedical relevance in the context of human genetic diseases $^{1,2}$ such as cystic fibrosis, multidrug resistance in various forms of cancer, and bacterial resistance to antibiotics. ${ }^{3}$

Whatever their function, $\mathrm{ABC}$ transporters share certain architectural requirements. First, they all have four domains: two transmembrane domains (TMDs) forming the ligandbinding site and two nucleotide-binding domains (NBDs) binding and hydrolyzing ATP to effect substrate translocation. The NBDs are highly conserved, suggesting a common transportdriving mechanism. They share several common motifs, including the Walker A and B motifs found in other ATPases and the $\mathrm{ABC}$ signature that is unique to the family. ${ }^{4}$ The TMDs, which additionally contain large cytosolic segments in ABC exporters, are less well conserved, possibly because of the diverse range of transported solutes. They comprise 8-20 membrane-spanning segments. In ABC importers the four domains exist on separate units, whereas bacterial exporters are half-transporters with one TMD and one NBD per polypeptide, functioning as homo- or heterodimers. Many eukaryotic ABC exporters comprise all four domains on a single polypeptide chain. Structural and genomic

\footnotetext{
† Université Libre de Bruxelles. E-mail: J.P.B., jpbecker@ulb.ac.be; M.P., mprevost@ulb.ac.be.

* Université catholique de Louvain. E-mail: F.V.B, vanbambeke@ facm. ucl.ac.be; P.M.T., tulkens@facm.ucl.ac.be.
}

analyses and TM predictions ${ }^{5}$ suggest that importers and exporters differ as regards both their TM domain organization and their topology.

The transport mechanism has been extensively studied, especially in ABC exporters, but it remains controversial. ${ }^{6}$ The catalytic cycle comprises ATP binding, formation of a NBD dimer sandwich, ATP hydrolysis, and dissociation of $\mathrm{P}_{\mathrm{i}}$ and ADP. The energy produced by this cycle is coupled to substrate translocation across the membrane. Two alternative models for this transport have been proposed, ${ }^{7}$ and the most significant difference is in the nature of the mechanism that drives the substrate from a high affinity to a low affinity site. In one model ${ }^{8}$ the formation of a closed NBD dimer provokes the conformational changes that are transmitted to the binding site and two sequential ATP hydrolysis events reset the protein. Other models ${ }^{9}$ require either one ${ }^{10}$ or two hydrolysis ${ }^{8}$ reactions to supply the efflux of the substrate and the resetting of the protein for another cycle.

At present, several X-ray structures of whole $\mathrm{ABC}$ transporters are available. All but one correspond to bacterial transporters. Five are ABC importers (BtuCD, ModBC-A, HI1470/1, MalFG/ $\mathrm{K}, \mathrm{MetNI}),{ }^{9-15}$ and three are drug exporters (SAV1866, MsbA, and mouse P-glycoprotein). ${ }^{16-19}$ High-resolution structures and NMR, EPR, and electron microscopy data strongly suggest that $\mathrm{ABC}$ exporters undergo large movements during substrate efflux, in particular upon ATP binding and/or hydrolysis. ${ }^{20-27}$ Although structure determinations and the large body of experimental data obtained on $\mathrm{ABC}$ transporters have shed light on the transport mechanism, the answers to some questions remain elusive: what are the conformational changes occurring upon substrate binding and ATP binding and/or hydrolysis, and how do these changes relate to solute translocation? 


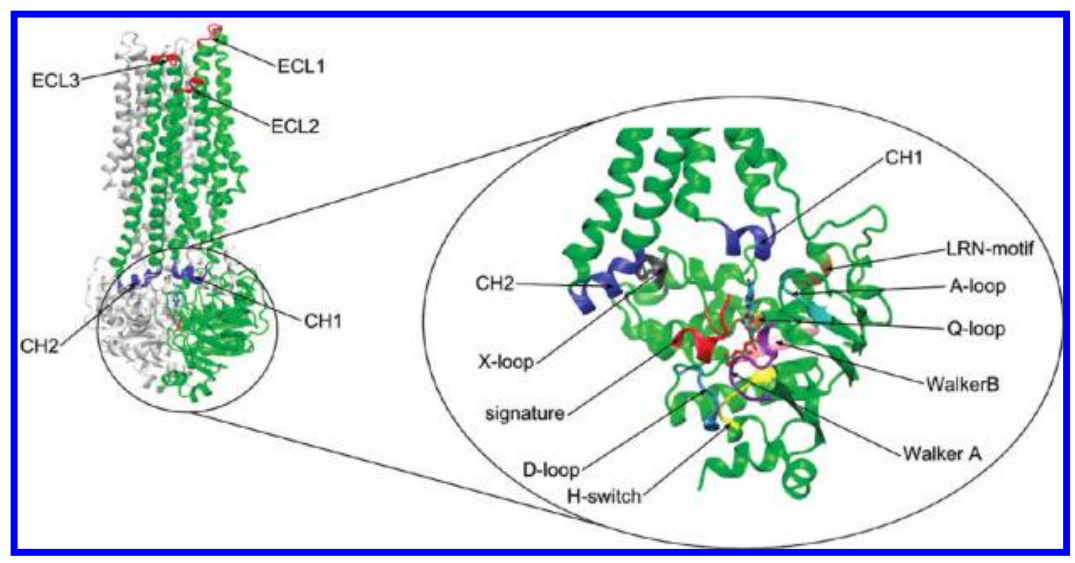

Figure 1. Ribbon representation depicting the crystal structure of the SAV1866 dimer (PDB code: 2ONJ). One monomer is green, the second monomer is white. The ATP molecules located in the NBDs are represented as sticks and are colored according to the atom represented (carbon in cyan, nitrogen in blue, oxygen in red, and phosphorus in bronze). ECL and CH stand for the extracellular loops and the intracellular coupling helices. The enlarged view shows a close-up illustration of one of the NBDs and of the main motifs common to members of the ABC exporter family.

Molecular dynamics (MD) simulations are a means of exploring the conformational dynamics of proteins with an extreme level of detail as regards atomic motions. The advent of increasingly powerful computers and improved simulation methodologies has made it possible to study trajectories spanning several dozen nanoseconds. Although this is still orders of magnitude less than the time required for major conformational changes $(\geq 100 \mathrm{~ms}$ for a transport event in P-glycoprotein, a eukaryotic $\mathrm{ABC}$ exporter ${ }^{28}$ ), $\mathrm{MD}$ simulations are expected to offer insights into the first steps that initiate different processes during the catalytic cycle. As determining membrane-protein structures remains a difficult task, almost unfeasible in the case of the wild-type protein complexed to its natural substrate, the MD approach can also fruitfully complement crystallographic analysis.

MD simulations have previously focused on the dynamic behavior of $\mathrm{ABC}$ importers. Trajectories of the monomeric and dimeric NBDs of MJ0796, ${ }^{29-32} \mathrm{HisP},{ }^{33}$ MalK, ${ }^{34}$ and $\mathrm{FbpC}^{35}$ have been analyzed upon ATP binding and/or hydrolysis to ADP. Recently, several simulations of the whole nucleotide-free and nucleotide-bound forms of the $\mathrm{BtuCD}^{36,37}$ and $\mathrm{BtuCDF}^{38}$ complexes and their components were performed to study the effect of ATP binding.

To the best of our knowledge, no conventional MD simulations of whole $\mathrm{ABC}$ exporters have yet been reported. A targeted MD simulation on MsbA transporter was recently published. ${ }^{39}$ SAV1866 is a homodimeric ABC "half-transporter". Its TMD, as in all known $\mathrm{ABC}$ bacterial exporters, contains six elongated helices extending into the cytoplasm (see Figure 1). SAV1866 structures have been determined in the presence of $\mathrm{ADP}^{16}$ and AMP-PNP, a nonhydrolyzable ATP analog. ${ }^{17}$ These two structures and the nucleotide-bound MsbA structures are very similar, featuring a drug extrusion chamber open toward the outside of the cell. They are believed to represent the outward-facing conformation in which the protein expels the substrate. In the present study we performed MD simulations, in the presence and in the absence of ATP/ $\mathrm{Mg}^{2+}$, starting from the nucleotidebound structure of SAV1866 embedded in an explicit bilayer. We used the trajectories to explore the dynamic behavior of SAV1866. Our aim was to learn what structural changes are caused by ATP binding and especially how the signal triggered by nucleotide binding is transmitted to the TMD.

\section{Methods}

Molecular Dynamics Simulations. Energy minimization, equilibration, and production dynamics runs for the two systems were performed with the Gromacs package v.3.3, ${ }^{40}$ starting from the crystallographic structure of SAV1866 complexed with AMP-PNP (PDB code: 2ONJ). AMP-PNP is an ATP analogue used to mimic an ATP-bound state.

The protein, lipids, and water interacted via the ffg $43 \mathrm{a} 2$ force field. Covalent bonds were constrained by means of the LINCS ${ }^{41}$ algorithm for the protein and SETTLE for water. ${ }^{42}$ This permitted the use of a 2 fs integration time step. Evaluation of van der Waals terms was done with a twin range cutoff of 1 and $1.4 \mathrm{~nm}$. To avoid truncation of long-range electrostatic interactions, the particle-mesh Ewald method ${ }^{43}$ was used.

To simulate the nucleotide-bound protein, an ATP molecule was built into each active site pocket, using the positions of the AMP-PNP molecules in the X-ray structure. The sodium ion located in the binding site of the X-ray structure was replaced with a magnesium ion. This construction was preferred to that of a docking of an ATP molecule in the binding pocket as carried out in a MD simulation study of BtuCD. ${ }^{44}$ According to Dawson and Locher ${ }^{17}$ the binding mode of AMP-PNP in SAV1866 is consistent with that of ATP in the NBD dimer of $\operatorname{Rad} 50^{45}$ and the electronic density corresponding to sodium ion is close to where magnesium and sodium ions were identified in isolated NBDs. ${ }^{45,46}$ Second, the ATP structure built from AMP-PNP in our system is bound in a similar fashion as in two other crystal structures, that of Hlyb (PDB code 1XEF) and of TAP1 (PDB code 2IXF). After superposition of the ATP of our system onto that in the Hlyb structure the root-meansquare deviation (RMSD) amounts to $1.1 \AA$, which given the resolution of the different full length structures cannot be considered as a high value. Moreover, the built $\mathrm{Mg}^{2+}$ interacts with similar residues as in the Hlyb and TAP1 structures. A protocol similar to ours was recently applied in a MD study of the conformational transition in MsbA. ${ }^{39}$

The system was then minimized for 100 steps, using the steepest descent algorithm to relieve bad crystal contacts. Next, the protein was inserted into a solvated lipid bilayer of 464 POPE, using a protocol developed by Kandt et al. ${ }^{47}$ The ATPbound simulation comprised the protein, 20 crystal water molecules, two $\mathrm{Mg}^{2+}$ ions, and two $\mathrm{Na}^{+}$ions solvated by 363 lipid and 41391 additional water molecules. The lipid bilayer 
and built water were first equilibrated for $10.8 \mathrm{~ns}$, using harmonic restraints on all the other atoms (proteins, ATP, $\mathrm{Mg}^{2+}$ ions, and $\mathrm{Na}^{+}$ions). A second independent simulation was set up by removing the ATP molecules and magnesium ions. The nucleotide-free simulation was carried out on a system consisting of the protein plus 20 crystal water molecules, $2 \mathrm{Na}^{+}$ions, and two $\mathrm{Cl}^{-}$ions solvated by 363 built lipids and 41435 built water molecules.

For both systems, unrestrained thermalization and equilibrations were then performed for 5.6 ns with a Berendsen thermostat $^{48}$ at $310 \mathrm{~K}$ and a barostat at 1 bar followed by $15 \mathrm{~ns}$ performed with a Nosé-Hoover thermostat ${ }^{49}$ at $310 \mathrm{~K}$ and a Parrinello-Rahman barostat at 1 bar. ${ }^{50}$ Production runs were performed for $100 \mathrm{~ns}$. Another simulation for both systems was carried out for $50 \mathrm{~ns}$ starting from the conformations obtained after the $15 \mathrm{~ns}$ equilibration of the first simulations.

To calculate the $\mathrm{p} K_{\mathrm{a}}$ values of residues potentially charged at $\mathrm{pH} 7$ in each system, a dielectric continuum model was used. A layer of spherical balls was built around the protein system to mimic the lipid bilayer. The UHBD program ${ }^{51}$ with an interface $^{52}$ was employed. Calculations were done assuming an internal dielectric of 20. The His 541 and His 571 residues were found to be protonated in both systems. In addition to these two histidines His 534 of the $\mathrm{H}$-switch motif was protonated in the nucleotide-bound protein only.

Analysis of the MD Trajectories. Principal component analysis (PCA) is a tool for identifying the essential subspace of large-scale collective fluctuations from MD trajectories or any set of structures. ${ }^{53,54}$ The method is based on calculation and diagonalization of the covariance matrix of atomic fluctuations, which yields a set of eigenvalues and eigenvectors. The eigenvectors of this matrix represent a set of directions in the $3 \mathrm{~N}$-dimensional space, along which the fluctuations observed in the simulation are uncoupled with respect to each other. The eigenvalues quantify the fluctuations along the respective eigenvectors. Thus the largest eigenvalues describe the dominant motions. The motions along the principal modes can be further analyzed by projecting the atomic trajectories onto the corresponding eigenvectors. The Gromacs program was used to analyze the two trajectories.

The dynamite server ${ }^{55}$ was used to compute correlated motions between residue pairs.

The residues participating in the NBD:NBD interface of the crystallographic structure of SAV1866 were identified with the PROTORP server. ${ }^{56}$

The MOLE program ${ }^{57}$ was used to detect access paths connecting the interior of the protein with its surrounding environment. Several conformations along the nucleotide-free and nucleotide-bound trajectories were monitored to analyze channels originating at the bottom center of the TM domains.

Hydrogen bonds, salt bridges, aromatic and ionic interactions, and contacts between residues of the protein were identified with the Gromacs package. Ionic interactions were defined as interactions between charged groups of charged residues at a distance ranging between 3.5 and $5 \AA$.

\section{Results}

Overall Conformational Changes. As a first check of the stability of the simulations and to assess quantitatively the extent of the motions, the root-mean-square deviation (RMSD) for all $\mathrm{C} \alpha$ positions of the protein was computed along the 100 and $50 \mathrm{~ns}$ trajectories of the production runs using the conformation at the start of the heating run for all simulations. In the $100 \mathrm{~ns}$ trajectories, for about the first $30 \mathrm{~ns}$, the RMSD computed on both trajectories was quite similar. The RMSD of the nucleotidefree simulation then departed from that of the nucleotide-bound trajectory and remained higher by about $0.5 \AA$ for the last 80 ns. For the 50 ns trajectories, the RMSDs are rather similar for the first $25 \mathrm{~ns}$. The RMSD of the nucleotide-free simulation then increases for about $10 \mathrm{~ns}$ to reach a difference of about $0.5 \AA$ during the last $15 \mathrm{~ns}$ relative to the RMSD of the nucleotide-bound simulation which remains steady. We also computed the RMSD for different domains of the protein (see Figure 2). A drift about $0.75 \AA$ larger was observed for the TMD dimer in the two nucleotide-free trajectories relative to their corresponding nucleotide-bound ones. For the NBD dimer, the RMSD was about the same between the nucleotide-free and nucleotide-bound system in the 100 and 50 ns trajectories. The superposition of TM1-TM2-TM3-TM6 segments revealed a larger drift for TM4-TM5 in the nucleotide-free trajectory, reaching about $1-1.5 \AA$. This finding is noteworthy, as in the different crystal structures of ABC exporters, the TM4-TM5 of one TMD crosses over and associates with the other monomer. This particular TM pair has been proposed to pivot around two hinges formed by the extracellular loops 2 (ECL2) and 3 (ECL3) (see Figure 1) so as to produce the conformational change from the outward- to the inward-facing structure. ${ }^{18}$

The flexibility of the protein was also analyzed by inspecting the root-mean-square fluctuation (RMSF) of each residue from its time-average position. The positional fluctuations of the $\mathrm{C} \alpha$ atoms were computed over the last $60 \mathrm{~ns}$ of the longer $100 \mathrm{~ns}$ production runs as the RMSD are rather steady during this time period for both the nucleotide-free and nucleotide-bound systems (Figure S1, Supporting Information). The fluctuations are larger for the two NBDs in the nucleotide-free simulation. The Walker $\mathrm{A}$, Walker $\mathrm{B}$, signature, and $\mathrm{X}-$ loop $^{16}$ displayed the largest differences in both monomers. In the TMDs the ECL2, the two cytosolic coupling helices $\mathrm{CH} 1$ and $\mathrm{CH} 2$ (short helical segments of the intracellular loops constituting the major interface of the TMDs with the NBDs; see Figure 1) and a large portion of TM4 showed larger fluctuations in the absence of the nucleotide. The RMSFs calculated for the $\mathrm{C} \alpha$ atoms of the nucleotide-bound simulation were compared to the B-factors from the crystallographic experiment. Qualitatively, the RMSFs agree well with the B-factors reproducing the alternation between the more rigid TM segments or secondary structures and the more flexible loops (see Figure S2, Supporting Information). In the simulation the TM domains feature larger fluctuations than the NBDs relative to the X-ray structure.

Transmembrane Chamber. The models proposed for the transport mechanism stress the importance of two discrete states of the transporter: the inward-facing conformation, prone to accommodate the substrate, and the outward-facing conformation that should facilitate its efflux. ${ }^{18,58-61}$ To detect a potential conformational response to the removal of $\mathrm{ATP} / \mathrm{Mg}^{2+}$, we therefore monitored the access paths or tunnels in several conformations selected along the $100 \mathrm{~ns}$ trajectories. As expected, the starting conformation, identical for both nucleotide-free and nucleotide-bound systems, displayed a large tunnel connecting the bottom of the TMD with the extracellular medium, as in the outward-facing crystal structure (see Figure 3 ). In the nucleotide-bound trajectory, this situation persisted for the whole $100 \mathrm{~ns}$. In the nucleotide-free trajectory, a central tunnel was still observed at $20 \mathrm{~ns}$, but with a much-reduced radius. An additional tunnel was found to depart from the central one and to exit between TM1 and TM2 near the middle of the membrane. In the nucleotide-free conformation at $40 \mathrm{~ns}$, the central tunnel appeared split in two near the middle of the 

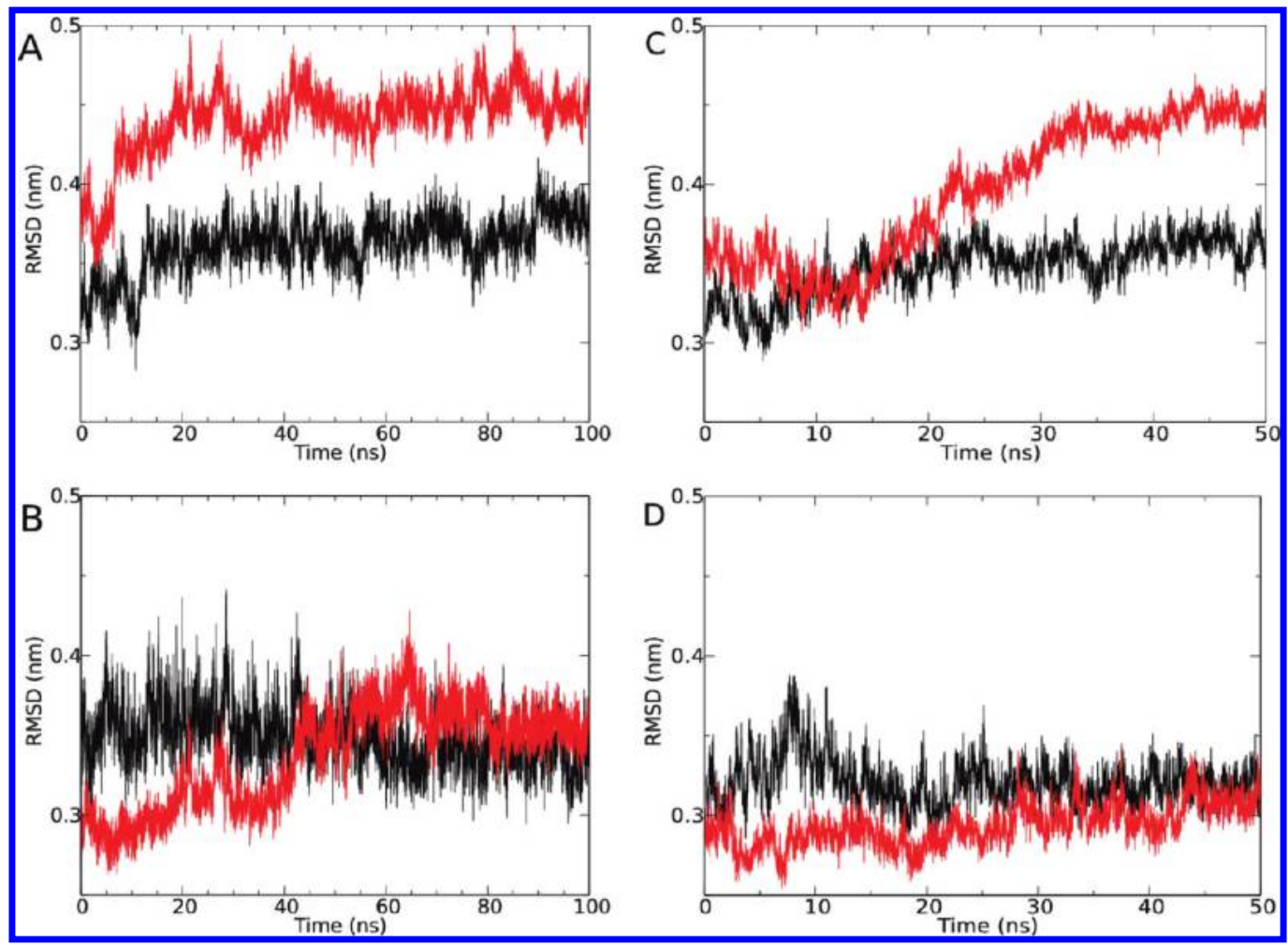

Figure 2. Root mean square deviation (RMSD) of the C $\alpha$ atoms of the TMDs (A) and the NBDs (B) of the 100 ns nucleotide-bound (black) and nucleotide-free (red) trajectories. (C) and (D) are computed over the $50 \mathrm{~ns}$ trajectories. The conformation at the start of the heating process was taken as the reference.

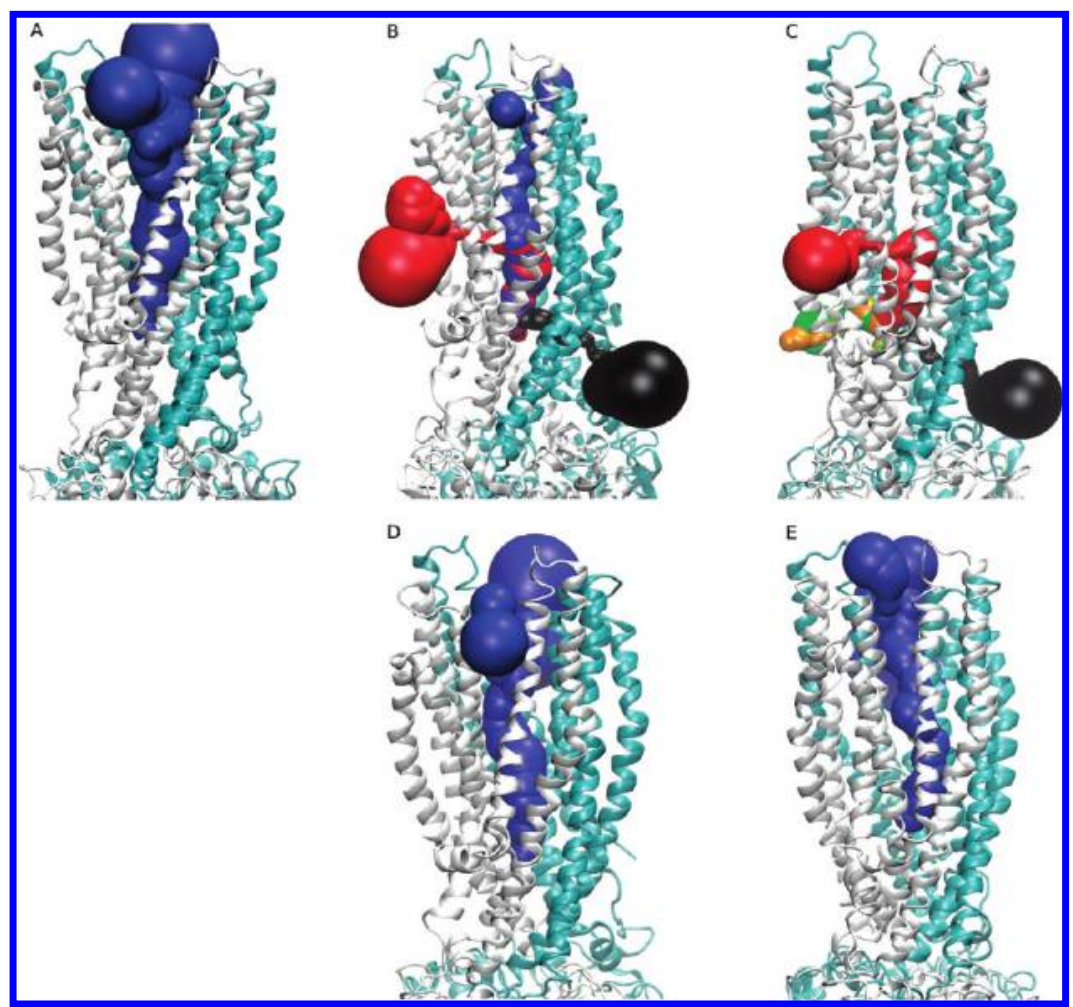

Figure 3. Dynamics of the internal TMD chamber in the nucleotide-free and the nucleotide-bound trajectories. Access paths originating at the bottom center of the TMD identified with the MOLE program ${ }^{57}$ at 0,40 , and $80 \mathrm{~ns}$ of the simulation time. Upper: In the nucleotide-free trajectory. (A) At $0 \mathrm{~ns}$ a central path is identified that eventually splits at its extracellular extremity. (B) At $40 \mathrm{~ns}$ a central path persists, albeit with a much smaller radius. (C) At $80 \mathrm{~ns}$, no central cavity exiting to the extracellular medium is identified anymore. Other, narrower paths exit to the cytosol and inner membrane leaflet. Lower: In the nucleotide-bound trajectory. (D) and (E) At 40 and $80 \mathrm{~ns}$ a central tunnel is observed. The 0 ns conformation is not shown as it is identical to that in the nucleotide-bound trajectory.

membrane (see Figure 3). This was predominantly due to the two TM6 segments getting closer to each other. Three additional, albeit much narrower, tunnels suggested ways out at the level of the cytosolic border and the inner leaflet of the membrane. 


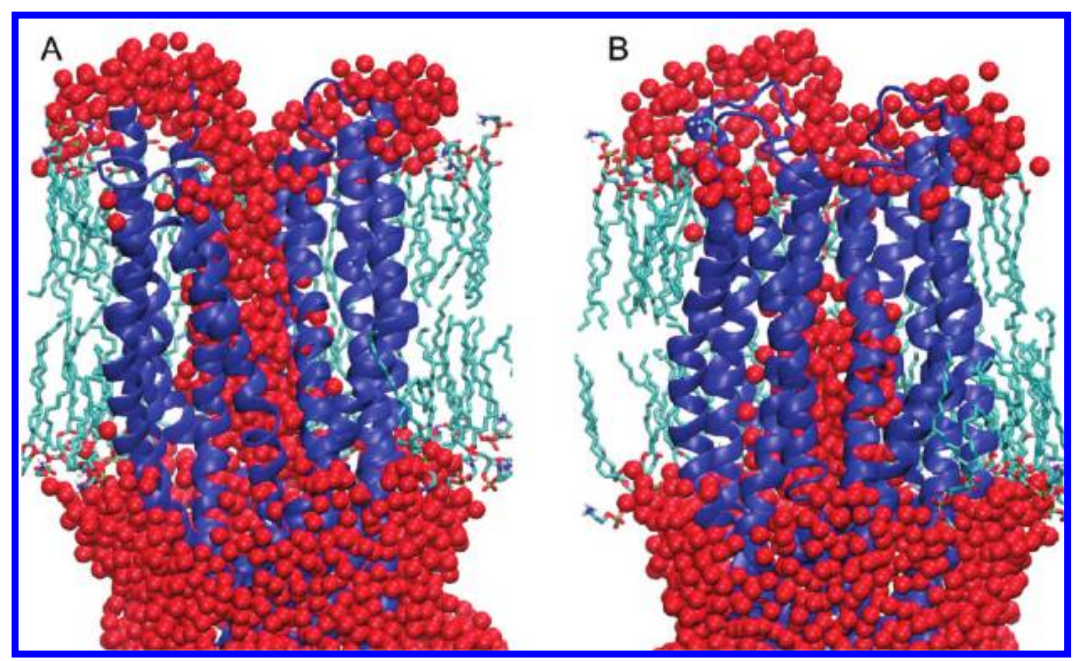

Figure 4. Snapshots of the conformation extracted at $80 \mathrm{~ns}$ from the nucleotide-bound (A) and nucleotide-free (B) trajectories. Dehydration of the internal chamber located in the TMD is observed in the nucleotide-free conformation at the level of the outer membrane leaflet. The protein is shown as blue ribbons, the water molecules as red spheres, and the lipids as sticks colored according to their chemical composition (carbon in cyan, nitrogen in blue, oxygen in red, and phosphorus in bronze).

The conformation at $80 \mathrm{~ns}$ revealed closure of the central chamber and did not feature any exit toward the extracellular medium (see Figure 3), but it still displayed tunnels exiting near the middle of the membrane and at the cytosol-membrane interface. The $50 \mathrm{~ns}$ trajectories show a similar behavior. At 50 ns the nucleotide-free conformation already features no exit of the central tunnel to the extracellular medium in contrast to the nucleotide-free trajectory (see Figure S3, Supporting Information).

The constriction of the central pore observed at $80 \mathrm{~ns}$ in the 100 ns nucleotide-free trajectory occurred over about $20 \AA$ from the middle of the membrane toward the extracellular side. When snapshots taken at $100 \mathrm{~ns}$ in the two simulations were superposed, closure of the TMD in the nucleotide-free trajectory was found to occur through a large motion of the outermembrane leaflet halves of the two TM6 regions and, to a lesser extent, through motions of the other TM segments. The distance between the TM6 N-terminal extremities (extracellular side) was found to decrease by $10-13 \AA$ in the nucleotide-free structure as compared to the nucleotide-bound conformation. Near the middle of the bilayer, in contrast, the centers of the two TM6 segments were found to be at most $1 \AA$ closer. The distance between the C-terminal extremities of TM6 on the cytosolic side was about $4 \AA$ greater in the nucleotide-free trajectory. These observations are in agreement with topographical analysis of TM6 ${ }^{62,63}$ in Pgp, indicating that the most ample displacements occur at the two ends of TM6.

The superposition also showed complete dehydration of the constricted region embedded in the outer membrane leaflet (Figure 4). The helix portions facing this region appeared to consist of about three helix turns of TM1, TM5, and TM6 in each monomer and to be composed solely of hydrophobic residues (TM1: Ile33, Ile37, Ile41 and Ile45; TM5: Ile260, Ile263, Ala267 and Ala270; TM6: Ile275, Leu280, Val284, and Leu287). The density profiles along the central tunnel axis averaged over the last $20 \mathrm{~ns}$ of the two trajectories confirmed depletion of water in the region affected by the constriction (Figure S4, Supporting Information). It is noteworthy that the TM1, TM5, and TM6 residues contributing to TMD constriction are exclusively hydrophobic and form a cluster upon closure. Interestingly, these hydrophobic residues appear to be rather well conserved across species. Except for the TM1 residues, all of them are shielded from the solvent in the nucleotide-bound trajectory and in the crystal structure. They become solventaccessible as a result of motions occurring during the nucleotidefree simulation. Solvent-exposed hydrophobic residues are expected to cause a destabilization that should promote both dehydration and hydrophobic collapse.

Monitoring the Interactions in the Nucleotide-Free and Nucleotide-Bound Simulations. The results described above suggest that removal of ATP/ $\mathrm{Mg}^{2+}$ from the NBDs induces closure of the TMD at the level of the outer membrane leaflet. To probe the mechanism of signal transmission from the ATPbinding pockets to the extracellular side of the TMDs, we analyzed how the absence of nucleotide modifies interactions in the nucleotide-free trajectory as compared to the nucleotidebound one. For this we identified the interactions (hydrogen bonds, ionic and aromatic interactions) involving $\mathrm{ATP} / \mathrm{Mg}^{2+}$ when present, the main NBD motifs, and $\mathrm{CH} 1, \mathrm{CH} 2$, representing the major interface of the TMDs with the NBDs. This was done in the $100 \mathrm{~ns}$ production runs as they correspond to the longest trajectories. The interactions whose persistence exceeded $50 \%$ over the last 50 ns are shown in Figure 5.

In the nucleotide-bound trajectory, as expected, the ATP/ $/ \mathrm{Mg}^{2+}$ ligands displayed numerous interactions with the Walker A, signature, Walker B, H-switch, and A-loop motifs. Interactions within each NBD appeared circumscribed to the Walker B-Hswitch and D-loop-signature motif pairs. Interactions were found to occur between the following motifs of opposite NBDs: H-switch-D-loop, Q-loop-Q-loop, Q-loop-X-loop and Q-loopsignature. The Q-loop and X-loop motifs also displayed a few interactions with $\mathrm{CH} 1$ and $\mathrm{CH} 2$ of the TMDs.

In the nucleotide-free trajectory, the motifs involved in interactions with $\mathrm{ATP} / \mathrm{Mg}^{2+}$ in the nucleotide-bound trajectory must turn around to find new partners. Within each NBD, Walker B-Walker A, H-switch-D-loop, and Walker B-H-switch interactions were identified. Between pairs of opposite NBDs, several $\mathrm{H}$-switch-H-switch, $\mathrm{H}$-switch-Walker $\mathrm{B}$ interactions were found. Interactions between the Q-loop, the X-loop, and the "LRN" motif, which is a previously unrecognized short sequence preceding the Q-loop that appears to be conserved in ABC exporters (see Figure 1 and Figure S5, Supporting Information) on the one hand and $\mathrm{CH} 1$ and $\mathrm{CH} 2$ on the other were found to have significantly increased relative to the nucleotide-bound trajectory. 


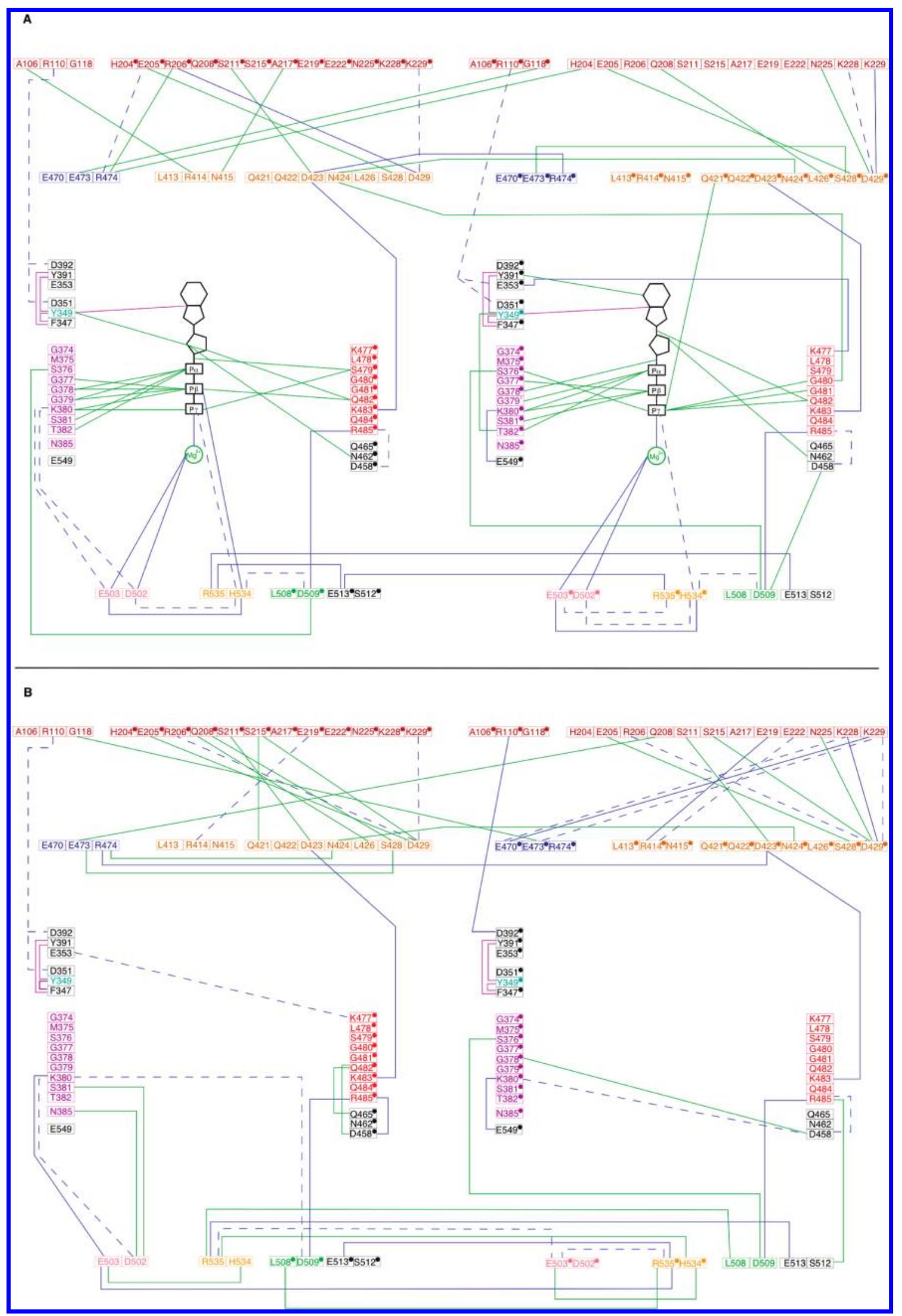

Figure 5. Interactions between $\mathrm{ATP} / \mathrm{Mg}^{2+}$, residues of the main NBD motifs, and the coupling helices $(\mathrm{CH})$ (see Figure 1) with a persistence higher than $50 \%$ during the last $50 \mathrm{~ns}(\mathrm{~A})$ in the nucleotide-bound and (B) in the nucleotide-free simulation. The colors of the lines indicate the nature of the interaction: hydrogen bond (green line), salt bridge (blue line), ionic interaction (dashed blue line), or aromatic-aromatic interaction (mauve). The boxed residues are colored according to the motif to which they belong (cyan, A-loop; mauve, Walker A; orange, Q-loop and "LRN" motif; blue, X-loop; red, signature; pink, Walker B; green, D-loop; yellow, H-loop; brown, CH). The $\bullet$ sign marks the residues of monomer 2. The 2D structure of ATP is represented in black lines and the magnesium ion is depicted as a green circle. 
Different experimental studies support the view that ATP binding powers formation of the NBD dimer. Most of the NBDs isolated to date are monomers, and addition of ATP or nonhydrolyzable ATP analogues does indeed drive dimerization of monomeric NBDs. ${ }^{64-67}$ Furthermore, several X-ray structures of $\mathrm{ABC}$ transporters ${ }^{13,17,18}$ determined in the absence and presence of nucleotide reveal significantly different separation distances between the NBDs.

All of these data led us to analyze the features of the NBD-NBD interface. First, we focused on the SAV1866 and MsbA nucleotide-bound X-ray structures determined in a closed dimer state (PDB codes 2ONJ and 3B60). In these structures, the interface is characterized respectively by 67 and $64 \%$ polar and charged residues. In comparison, mean values of 58 and $60 \%$ were observed in a survey of homodimers and transient dimers, respectively. ${ }^{68,69}$ These results suggest that the NBD-NBD interface in these two exporters is quite hydrophilic and charged as compared to other dimers. This could have some bearing on the phenomenon of association/dissociation of the NBD dimer upon ATP binding/hydrolysis. In the dissociated state, this interface is indeed expected to be partially or completely hydrated, which is consistent with a predominantly polar surface.

We then monitored various types of interactions at the NBD dimer interface. Over the last entire $100 \mathrm{~ns}$ time span, a higher total number of hydrogen bonds was formed between residues of NBD1 and NBD2 in the nucleotide-bound trajectory than in the nucleotide-free trajectory (Figure S6, Supporting Information). In addition, the number of hydrogen bonds featuring a persistence time superior or equal to $25 \%$ over the last $20 \mathrm{~ns}$ was one-third higher in the nucleotide-bound trajectory. We also computed the number of contacts made between residues in NBD1 and NBD2. As in the case of the hydrogen bonds, the number of atomic contacts was found to be greater in the nucleotide-bound trajectory (data not shown). These findings indicate that the NBD dimer is more loosely packed in the nucleotide-free trajectory.

These data, together with the polar character of this interface, prompted us to examine whether water molecules had penetrated the NBD-NBD interface along the trajectories. We thus searched for water molecules forming hydrogen bonds with residues of the NBD interface during the last $20 \mathrm{~ns}$, with a persistence exceeding $25 \%$. The average number of water molecules meeting these criteria was 79 for the nucleotide-free trajectory and 30 for the nucleotide-bound trajectory. The nucleotide-free interface is thus clearly more hydrated (Figure S7, Supporting Information), the most strongly hydrated motifs being the D-loop, Walker A, Walker B, signature, X-loop, and Q-loop motifs. This last is the most hydrated of all.

An examination of the residues to which these internal water molecules hydrogen bond revealed the pathways that they follow. In the nucleotide-free trajectory, all water molecules were found to enter the interface by hydrogen bonding with surface residues lining the entrance to the ATP-binding sites, among them D509, A507, D458, N455, and R485. They appeared subsequently to enter the ATP-binding pocket and to hydrogen bond to the residues of the main motifs (D-loop, Walker A, Walker B, signature, X-loop). Some of them were found to penetrate more deeply into the NBD, coming in contact with the Q-loop and reaching $\mathrm{CH} 1$ and $\mathrm{CH} 2$. The water molecules exiting the protein interior over this $20 \mathrm{~ns}$ period were found to follow roughly the same path as the one they took to enter.

The nucleotide-bound trajectory showed quite a different hydration pattern (Figure S7, Supporting Information). Of the
12 identified water molecules buried in the NBD dimer interface, six turned out to be crystal water molecules, three of which were found to hydrate the top of each ATP-binding site as in the X-ray structure. In NBD1, these three water molecules were found to H-bond to the Q-loop, the signature, the A-loop that stacks adenine, Y391 that itself stacks the A-loop, and to a lesser extent, the Walker A motif. The situation appeared almost symmetrical in NBD2. The six noncrystal water molecules were found to hydrate almost the same protein regions: the Q-loop, the signature, the Walker A residues except for the aromatic residues involved in stacking interactions. One of these six water molecules was seen to exit the protein interior via the X-loop and residues downstream from the $\mathrm{N}$-terminal Q-loop (residues 428 and 429).

Collective Motions. Proteins often accomplish their functions through collective atomic motions. To examine which residues undergo concerted motions, we analyzed the covariance matrix derived from the sets of conformations generated in the last 60 ns of the $100 \mathrm{~ns}$ simulations. This analysis highlighted regions of the protein that move together. Aside from anticipated correlations between the motions of secondary structural elements and those of residues near them in the sequence, a few residue motions showing $70 \%$ correlation were identified in the nucleotide-free trajectory (Figure S8, Supporting Information). Among them, the motions of residues $305-311$ in the TM6 C-terminal half were found to correlate with motions of residues 185-190 of the TM4 C-terminus and residues 127, 130, and 131 in the N-terminal part of TM3. Residues in the TM6 $\mathrm{N}$-terminal half $(279,282,283,285$, and 286) showed motions correlating with those of residues 34 and 38 of the TM1 C-terminus and 160 of the TM3 C-terminus. In the second monomer, the motions of residues $305-311$ of the TM6 C-terminus showed correlations with those of residues 126-134 of TM3 and residues $182-191$ of TM4, while correlated motions were also observed between residues 278 and 282 of the TM6 $\mathrm{N}$-terminus and residues 31 and 41 of TM1. No equivalent correlated motions were observed in the nucleotide-bound trajectory.

Principal component analysis over the last $60 \mathrm{~ns}$ of the 100 ns trajectories was performed to isolate global concerted motions from local fluctuations. This was done by concatenating the two trajectories fitted onto a same reference structure, from which a covariance matrix was constructed. Upon diagonalization of this matrix, five vectors represented $80 \%$ of the fluctuations. The similarities (or differences) and overlap of the sampled phase space were analyzed by projection of each separate simulation onto the plane defined by different pairs of eigenvectors taken among the first ten (data not shown). No overlap between the two trajectories was observed along eigenvector 1 (Figure S9, Supporting Information). On the average, the two trajectories displayed different positions along eigenvector 1 (Figure S10, Supporting Information). The comparison between the starting and the final conformations along eigenvector 1 reveals a large swing that brings the TM3-TM4-TM5-TM6 segments onto those in the MsbA inward-facing structure (PDB code $3 \mathrm{~B} 5 \mathrm{X})$ in the half outer membrane leaflet of the TMDs (see Figure S11, Supporting Information). This conformational change can be pictured as a leverage effect in which a slight displacement at the cytosolic side produces a much larger one at the extracellular side.

Visualization of the displacements along this eigenvector (Figure 6), which represents about $65 \%$ of the fluctuation revealed a first principal motion corresponding to constriction of the pore and characterized by a large displacement of the 


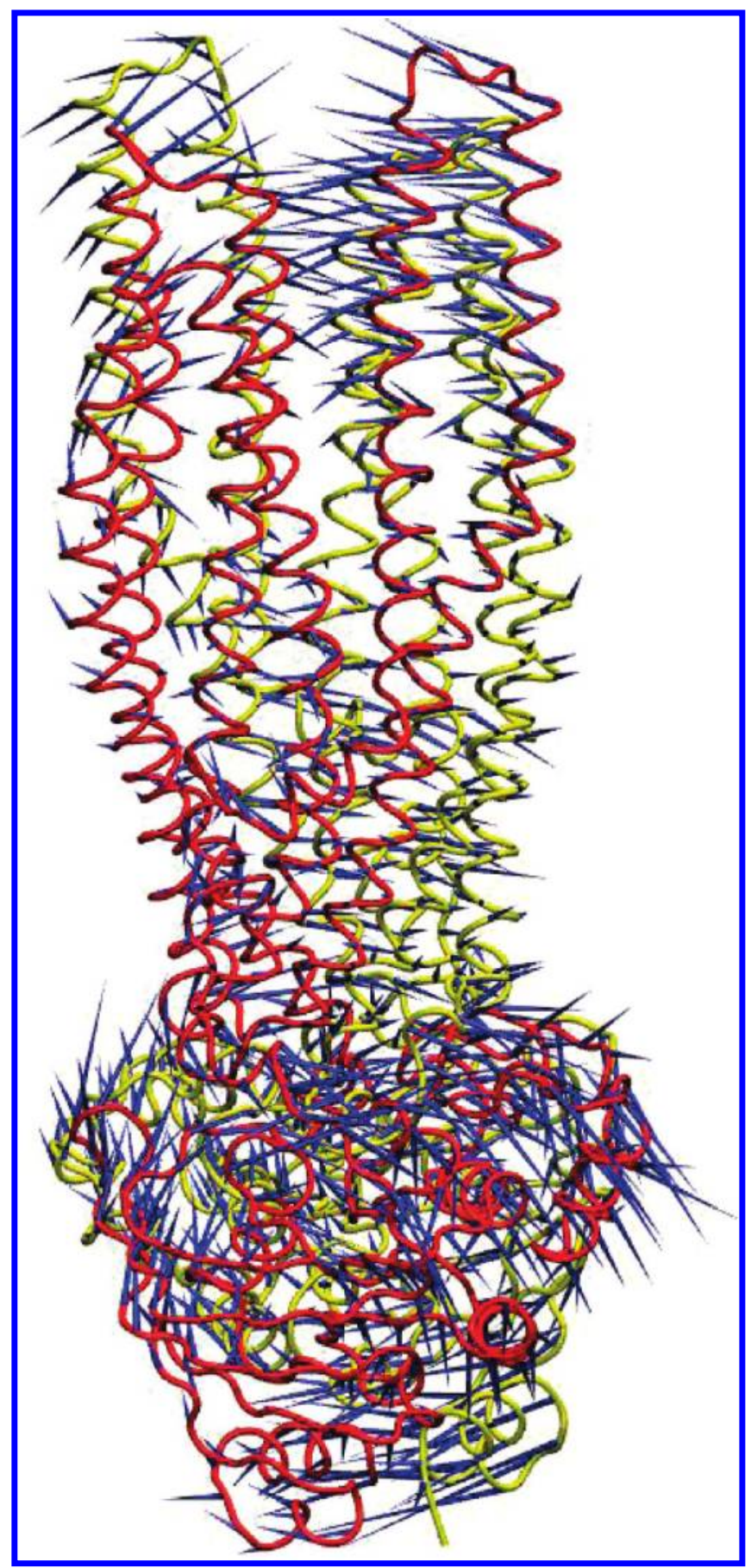

Figure 6. Porcupine plot of the motion corresponding to the first eigenvector defined on the basis of the combined trajectories. Each $\mathrm{C} \alpha$ atom has a blue cone attached to it, pointing in the direction of motion described by the eigenvector for this atom. The protein is depicted as a tube. One monomer is colored in red, the other in yellow.

outer membrane leaflet portion of TM6 and a lesser displacement of TM1 and TM5 in each monomer. The coupling helices, particularly $\mathrm{CH} 2$, and the cytosolic side of TM6, also appeared to undergo large displacements. Concomitantly, a global rotation of both NBDs, in opposite directions, was observed.

Proposed Transmission Mechanism. Our simulations show that the major change upon removal of $\mathrm{ATP} / \mathrm{Mg}^{2+}$ from its binding pocket in the cytosolic domain is closure of the TMD on the extracellular side. This is remarkable, as the residues involved in closure are located far from the ATP-binding pockets. This suggests that a signal must be transmitted from the NBDs all the way to the TMDs, as several experimental studies suggest. ${ }^{7,60,72,73}$ A question remaining to be answered is, how does the signal caused by ATP/ $\mathrm{Mg}^{2+}$ removal from the NBDs propagate and promote structural changes in the TMDs? According to the analysis of the MD trajectories, one suggests that the mechanism for transmission changes can take place following the series of events: The absence of nucleotide engenders two major local changes. First, new polar and charged interactions, involving motifs of the binding pockets, substitute for the lost interactions formed with ATP/ $\mathrm{Mg}^{2+}$. Second, the empty ATP binding pockets quickly fill with water molecules. The signal upon ATP removal is likely to be transmitted through the NBD-TMD interface. Persistent noncovalent interactions between the Q-loop, the X-loop, and the "LRN" motifs on one hand and the $\mathrm{CH} 1$ and $\mathrm{CH} 2$ coupling helices on the other hand occur in a significantly larger number at the NBD-TMD interface in the nucleotide-free trajectory. This observation sustains the functional importance of these motifs mentioned in other studies. ${ }^{16,38}$ Water molecules that entered via the nucleotide binding pockets travel through the NBD-NBD interface to the NBD-TMD interface. The role of this increased hydration is not clear. Water has been proposed to serve as a lubricant favoring the folding/unfolding process of proteins. ${ }^{72}$ A similar role could be conceived for water in the association/ dissociation process of proteins. These changes at the NBD-TMD interface must propagate to the TMD at the extracellular side. Our analysis of correlated motions reveals that, in the nucleotidefree trajectory, motions of residues of TM3 and TM4 connected to $\mathrm{CH} 1$ and $\mathrm{CH} 2$ highly correlate with those of the cytosolic residues of TM6. This triggers a motion as in a lever arm and causes a displacement of TM6 together with the other TM segments at the extracellular side leading to TMD closure.

Closure of the TMD at the extracellular side is clearly seen in the crystal structures of P-gp and MsbA determined in the absence of nucleotide. In these structures, however, closure is accompanied by an opening at the cytosol side. We do not see such an opening here (see Figure 2 and Figure S7, Supporting Information), although the NBD dimer is more loosely formed in the nucleotide-free trajectory and the interface significantly more hydrated. The absence of dimer dissociation may be due to the limited time scale accessible by conventional MD simulations. Closure of the TMD favored by transient exposure of hydrophobic residues may constitute a first step before a more complete dimer separation and the appearance of fully hydrated NBD monomers occur. The changes observed in our trajectories may thus constitute the initial steps of nascent large conformational changes occurring upon nucleotide binding. Notably, the quasi absence of a NBD dimer separation has also been observed in $\mathrm{MD}$ simulations of BtuCD, a full $\mathrm{ABC}$ importer. Only simulations of isolated BtuD displayed a closure upon ATP binding. ${ }^{37}$

Our simulation data thus propose that the TMD closure at the extracellular side occurs on a faster scale that the NDB dimer separation and, furthermore, that this TMD motion may occur without a large disengagement of the NBD monomers. Experiments on isolated NBDs show that the latter event may indeed occur in the absence of any TMD. What about the potential closure/aperture of the TMDs taking place in the absence of the NBD? One experiment performed on a truncated LmrA protein containing the TMD but lacking the NBDs that was shown to retain the substrate specificity of the full-length protein and mediated drug influx ${ }^{73}$ suggests that such a conformational change could occur. All these data hint that complete NDB separation could not have to be concomitant to TMD. 
Despite the fact that only two MD simulations were performed, one long and one shorter, several observations in the conformational dynamics of our simulations have possible mechanistic implications. In summary, our results suggest that removal of ATP/ $\mathrm{Mg}^{2+}$ from the binding pocket induces changes in interactions and amplified hydration in the NBDs, spreading from the Q-loop and X-loop toward the NBD-TMD interface and particularly to the coupling helices $\mathrm{CH} 1$ and $\mathrm{CH} 2$. These changes trigger highly correlated motions between the TM3 and TM4 helices, connected to $\mathrm{CH} 1$ and $\mathrm{CH} 2$, respectively, and TM6. Motions on the cytosolic side of TM6 spread over its outer membrane leaflet portion and over other neighboring helices to promote closure of the TMD on the extracellular side. Several studies have stressed the pivotal importance of TM6 in P-gp during the drug transport process ${ }^{62,63,74,75}$ and its participation in bidirectional communication between the TMDs and the NBDs in coupled transport. A recent study used targeted MD simulations of MsbA to explore the conformational transition from the outward-facing to the inward-facing states of the protein. It revealed a clear breaking of the NBD dimer interface in contrast to our study. ${ }^{39}$ The targeted MD method has the advantage of allowing an exploration of slow conformational transitions which are impossible to reach by conventional MD. However, in targeted MD all or a subset of atoms are guided toward a final target structure by means of a steering force and the generated trajectories may not always follow the lowest free energy path. Despite these differences our conventional and this targeted MD simulations have in common to point to the importance of the X-loop motif in the cross-talk between the NBDs and the TMDs and to the role of TM6 in the transmission of a large motion at the extracellular side and its correlated motions with TM3 and TM4.

These results also stress the importance to perform additional MD simulations ${ }^{38,76,77}$ of even longer time scale to possibly confirm these observations.

Acknowledgment. M.P. and F.V.B. are Maîtres de Recherche of the Belgian Fonds de la Recherche Scientifique (F.R.S.-FNRS). J.P.B. is a postdoctoral fellow supported by the F.R.S.-FNRS. This work was supported by the Belgian Fonds pour la Recherche Scientifique Médicale (grant no. 3.4583.08). We thank Julien Dupont for his technical assistance.

Supporting Information Available: Several properties calculated from the MD trajectories are shown (RMSF, access paths, density profile). The sequence alignment shows the conservation of a new revealed "LRN" motif. The detailed results of the principal component analysis and correlated motions are shown. This material is available free of charge via the Internet at http://pubs.acs.org.

\section{References and Notes}

(1) Dean, M.; Rzhetsky, A.; Allikmets, R. Genome Res. 2001, 11, 1156 (2) Gottesman, M. M.; Ambudkar., S. V. J. Bioenerg. Biomembr. 2001, $33,453$.

(3) Leonard, G. D.; Fojo, T.; Bates, S. E. Oncologist 2003, 8, 411.

(4) Jones, P. M.; O'Mara, M. L.; George, A. M. Trends Biochem. Sci. 2009, 34, 520 .

(5) Davidson, A. L.; Dassa, E.; Orelle, C.; Chen, J. Microbiol. Mol. Biol. Rev. 2008, 72, 317.

(6) Seeger, M. A.; van Veen, H. W. BBA-Proteins Proteom. 2009, 1794, 725 .

(7) Higgins, C. F.; Linton, K. J. Nat. Struct. Mol. Biol. 2004, 11, 918 (8) Sauna, Z. E.; Kim, I. W.; Ambudkar, S. V. J. Bioenerg. Biomembr. 2007, 39, 481 .

(9) Locher, K. P.; Lee, A. T.; Rees, D. C. Science 2002, 296, 1091
(10) Hvorup, R. N.; Goetz, B. A.; Niederer, M.; Hollenstein, K.; Perozo, E.; Locher, K. P. Science 2007, 317, 1387.

(11) Hollenstein, K.; Frei, D. C.; Locher, K. P. Nature 2007, 446, 213.

(12) Gerber, S.; Comellas-Bigler, M.; Goetz, B. A.; Locher, K. P. Science 2008, 321, 246.

(13) Pinkett, H. W.; Lee, A. T.; Lum, P.; Locher, K. P.; Rees, D. C. Science 2007, 315, 373 .

(14) Oldham, M. L.; Khare, D.; Quiocho, F. A.; Davidson, A. L.; Chen, J. Nature 2007, 450, 515.

(15) Kadaba, N. S.; Kaiser, J. T.; Johnson, E.; Lee, A.; Rees, D. C. Science 2008, 321, 250.

(16) Dawson, R. J. P.; Locher, K. P. Nature 2006, 443, 180.

(17) Dawson, R. J. P.; Locher, K. P. FEBS Lett. 2007, 581, 935.

(18) Ward, A.; Reyes, C. L.; Yu, J.; Roth, C. B.; Chang, G. Proc. Natl. Acad. Sci. U.S.A. 2007, 104, 19005.

(19) Aller, S. G.; Yu, J.; Ward, A.; Weng, Y.; Chittaboina, S.; Zhuo, R. P.; Harrell, P. M.; Trinh, Y. T.; Zhang, Q. H.; Urbatsch, I. L.; Chang, G. Science 2009, 323, 1718.

(20) Loo, T. W.; Bartlett, M. C.; Clarke, D. M. Biochemistry 2007, 46, 9328.

(21) Lee, J. Y.; Urbatsch, I. L.; Senior, A. E.; Wilkens, S. J. Biol. Chem. 2008, 283, 5769 .

(22) Dong, J. H.; Yang, G. Y.; Mchaourab, H. S. Science 2005, 308, 1023.

(23) Borbat, P. P.; Surendhran, K.; Bortolus, M.; Zou, P.; Freed, J. H.; Mchaourab, H. S. PLoS Biol. 2007, 5, 2211.

(24) Ward, A.; Mulligan, S.; Carragher, B.; Chang, G.; Milligan, R. A. J. Struct. Biol. 2009, 165, 169.

(25) Zou, P.; Mchaourab, H. S. J. Mol. Biol. 2009, 393, 574.

(26) Zou, P.; Bortolus, M.; Mchaourab, H. S. J. Mol. Biol. 2009, 393, 586.

(27) van Veen, H. W.; Margolles, A.; Muller, M.; Higgins, C. F.; Konings., W. N. EMBO J. 2000, 19, 2503.

(28) Ambudkar, S. V.; Cardarelli, C. O.; Pashinsky, I.; Stein, W. D. J. Biol. Chem. 1997, 272, 21160.

(29) Campbell, J. D.; Sansom, M. S. P. FEBS Lett. 2005, 579, 4193. (30) Jones, P. M.; George, A. M. J. Biol. Chem. 2007, 282, 22793.

(31) Jones, P. M.; George, A. M. Proteins 2009, 75, 387.

(32) Oliveira, A. S.; Baptista, A. M.; Soares, C. M. J. Phys. Chem. B 2010, 114, 5486.

(33) Campbell, J. D.; Deol, S. S.; Ashcroft, F. M.; Kerr, I. D.; Sansom, M. S. P. Biophys. J. 2004, 87, 3703.

(34) Wen, P. C.; Tajkhorshid, E. Biophys. J. 2008, 95, 5100.

(35) Newstead, S.; Fowler, P. W.; Bilton, P.; Carpenter, E. P.; Sadler, P. J.; Campopiano, D. J.; Sansom, M. S. P.; Iwata, S. Structure 2009, 17, 1213.

(36) Tanizaki, S.; Feig, M. J. Phvs. Chem. B 2006, 110, 548.

(37) Sonne, J.; Kandt, C.; Peters, G. H.; Hansen, F. Y.; Jensen, M. O.; Tieleman, D. P. Biophvs. J. 2007, 92, 2727.

(38) Ivetac, A.; Campbell, J. D.; Sansom, M. S. P. Biochemistry 2007, 46, 2767.

(39) Weng, J.-W.; Fan, K.-N.; Wang, W.-N. J. Biol. Chem. 2010, 285, 3053.

(40) Lindahl, E.; Hess, B.; van der Spoel, D. J. Mol. Model. 2001, 7, 306.

(41) Hess, B.; Bekker, H; Berendsen, H. J. C.; Fraaije, J. G. E. M. J. Comput. Chem. 1997, 18, 1463.

(42) Miyamoto, S.; Kollman, P. A. J. Comput. Chem. 1992, 13, 952.

(43) Darden, T.; York, D.; Pedersen, L. J. Chem. Phvs. 1993, 98, 10089.

(44) Oloo, E. O.; Tieleman, P. D. J. Biol. Chem. 2004, 279, 45013.

(45) Hopfner, K. P.; Karcher, A.; Shin, D. S.; Craig, L.; Arthur, L. M.; Carney, J. P.; Tainer, J. A. Cell 2000, 101, 789.

(46) Smith, P. C.; Karpowich, N.; Millen, L.; Moody, J. E.; Rosen, J.; Thomas, P. J.; Hunt, J. F. Mol. Cell 2002, 10, 139.

(47) Kandt, C.; Ash, W. L.; Tieleman, D. P. Methods 2007, 41, 475.

(48) Berendsen, H. J. C.; Postma, J. P. M.; van Gunsteren, W. F.; DiNola,

A; Haak, J. R. J. Chem. Phvs. 1984, 81, 3684.

(49) Hoover, W. G. Phys. Rev. A 1985, 31, 1695.

(50) Parrinello, M.; Rahman, A. J. Appl. Phvs. 1981, 52, 7182.

(51) Madura, J. D.; Briggs, J. M.; Wade, R. C.; Davis, M. E.; Luty,

B. A.; Ilin, A.; Antosiewicz, J.; Gilson, M. K.; Bagheri, B.; Scott, L. R.;

McCammon, J. A. Comput. Phys. Commun. 1995, 91, 57.

(52) Schaefer, M.; Bartels, C.; Karplus, M. J. Mol. Biol. 1998, 284, 835.

(53) Amadei, A.; Linssen, A. B. M.; Berendsen, H. J. C. Proteins 1993, $17,412$.

(54) Ortmans, I.; Prévost, M. J. Phvs. Chem. B 2008, 112, 8730.

(55) Barrett, C. P.; Hall, B. A.; Noble, M. E. M. Acta Crystallogr D 2004, 60, 2280.

(56) Reynolds, C.; Damerell, D.; Jones, S. Bioinformatics 2009, 25, 413.

(57) Petrek, M.; Kosinova, P.; Koca, J.; Otyepka, M. Structure 2007, $15,1357$.

(58) Jardetzky, O. Nature 1966, 211, 969. 
(59) Ramachandra, M.; Ambudkar, S. V.; Chen, D.; Hrycyna, C. A.; Dey, S.; Gottesman, M. M.; Pastan, I. Biochemistry 1998, 37, 5010. (60) Rosenberg, M. F.; Velarde, G.; Ford, R. C.; Martin, C.; Berridge,

G.; Kerr, I. D.; Callaghan, R.; Schmidlin, A.; Wooding, C.; Linton, K. J.; Higgins, C. F. $E M B O$ J. 2001, 20, 5615.

(61) Loo, T. W.; Clarke, D. M. Proc. Natl. Acad. Sci. U.S.A. 2002, 99 3511 .

(62) Storm, J.; Modok, S.; O’Mara, M. L.; Tieleman, D. P.; Kerr, I. D.; Callaghan, R. Biochemistry 2008, 47, 3615.

(63) Rothnie, A.; Storm, J.; Campbell, J.; Linton, K. J.; Kerr, I. D.; Callaghan, R. J. Biol. Chem. 2004, 279, 34913.

(64) Kerr, I. D. BBA-Biomembranes 2002, 1561, 47.

(65) Moody, J. E.; Millen, L.; Binns, D.; Hunt, J. F.; Thomas, P. J. J. Biol. Chem. 2002, 277, 21111.

(66) Sauna, Z. E.; Ambudkar, S. V. Mol. Cancer Ther. 2007, 6, 13. (67) Sauna, Z. E.; Kim, I. W.; Nandigama, K.; Kopp, S.; Chiba, P.; Ambudkar, S. V. Biochemistry 2007, 46, 13787.

(68) Nooren, I. M.; Thornton, J. M. J. Mol. Biol. 2003, 325, 991.
(69) Nooren, I. M. A.; Thornton, J. M. EMBO J. 2003, 22, 3486.

(70) Loo, T. W.; Bartlett, M. C.; Clarke, D. M. J. Biol. Chem. 2003, $278,1575$.

(71) Loo, T. W.; Bartlett, M. C.; Clarke, D. M. Biochemistry 2005, 44, 10250.

(72) Priev, A.; Almagor, A.; Yedgar, S.; Gavish, B. Biochemistry 1996, 35, 2061.

(73) Venter, H.; Shilling, R. A.; Velamakanni, S.; Balakrishnan, L.; van Veen, H. W. Nature 2003, 426, 866.

(74) Ma, J. F.; Géraldine, G.; Melera, P. W. Mol. Pharmacol. 1997, 51, 922.

(75) Loo, T. W.; Clarke, D. M. J. Biol. Chem. 1997, 272, 20986.

(76) Kandt, C.; Tieleman, D. P. Proteins 2010, 78, 738.

(77) Aittoniemi, J.; de Wet, H.; Ashcroft, F. M.; Sansom, M. S. $\underline{P l o S}$ Comput. Biol. 2010, 6, e 10000762.

JP1038392 